\title{
ARTE DIGITAL MX
}

\author{
Cynthia Patricia Villagómez Oviedo \\ Universidad de Guanajuato
}

\begin{abstract}
Resumen
El arte digital es poco conocido en México, si bien hay artistas digitales y algunos centros de exhibición como el Laboratorio Arte Alameda o el Centro Nacional de la Artes, dichos centros se encuentran en la capital del país, fuera de ésta continúan imperando las expresiones tradicionales del arte, como la pintura, la escultura y el grabado. Por lo que en este artículo analizamos los procesos de creación de artistas digitales mexicanos, de tal manera que sea posible proveer a otros artistas de métodos de desarrollo de ideas nuevas y originales, así como generar conocimiento que facilite la comprensión de los trabajos digitales, contribuyendo así al crecimiento, difusión y desarrollo del Arte digital mexicano. A la par se indaga sobre los procesos de reflexión y conceptualización en el trabajo artístico, desde el punto de vista del proceso intelectual llevado a cabo por el artista, reflejo de los nuevos procesos de creación de los artistas digitales mexicanos y sus fuentes de inspiración.
\end{abstract}

\section{Palabras-clave: ARTE DIGITAL; CREACIÓN ARTÍSTICA; PROCESOS ARTÍSTICOS; ARTISTAS DIGITALES MEXICANOS; METODO- LOGÍA}

\section{DIGITAL ART MX}

\section{Abstract}

Digital art is little known in Mexico, there are digital artists and some institutes which exhibit Digital art, like Laboratorio Arte Alameda and Centro Nacional de la Artes, these centers are located in Mexico City. But outside Mexico City, traditional art expressions: painting, sculpture, engraving are still the most important. So in this article we analyse creation processes of Mexican digital artists, to provide other digital artists with methods of development of original and new ideas, so as to provide knowledge that facilites comprehention of Digital art work and to contribute to the development of Digital art. We also research on the processes of artistic creation, which reflects the new processes of creation of Mexican digital artists and their inspiration.

\section{Keywords: DIGITAL ART;ARTISTIC CREATION; ARTISTIC PROCESSES; MEXICAN DIGITAL ARTISTS; METHODOLOGY}

Villagómez Oviedo, Cynthia Patricia. "Arte Digital MX". AusArt 6 (1):
285-295. D0I: 10.1387/ausart.19384

\section{AUSART}




\section{INTRODUCCIÓN}

El presente trabajo gira en torno a los procesos de producción de artistas digitales mexicanos y su obra, en el entendido que, comúnmente, estos procesos están envueltos por un halo de misterio: los procesos de creación son desconocidos y las más de las veces solo es posible ver la obra terminada. De ahí la necesidad de indagar sobre estos procesos, en el entendido de que, en México, a la fecha, no se ha realizado investigación a este respecto. Se parte de la idea de que es posible detectar los procesos de producción de ideas en los artistas digitales mexicanos, a través de la observación no sólo de los propios procesos, sino también de su contexto, de tal manera que se pueda proveer a otros artistas de nuevos métodos de desarrollo de ideas originales y nuevas, así como generar conocimiento que facilite la comprensión de trabajos digitales, contribuyendo al crecimiento, difusión y desarrollo del Arte digital mexicano.

\section{EL PROCESO DE CREACIÓN}

El Arte Digital (en adelante AD) agrupa todas aquellas manifestaciones artísticas llevadas a cabo mediante un ordenador. Son obras de arte que pueden ser realizadas con medios digitales y que pueden ser reducidas a una serie electrónica de unos y ceros (Lieser 2009). Una obra de este tipo es producida por creadores que usan medios computacionales en los pasos previos a la materialización de la obra o en su exhibición (Giner 2007). El AD combina Arte, Ciencia y Tecnología. Aunque no todo lo producido con estos recursos es $A D$, se puede decir que la frontera es imprecisa. Pero debido a esto el $A D$ es semillero de nuevas ideas, por lo que la investigación en torno a sus procesos de creación es indispensable.

Aquí vale la pena preguntarnos si realmente existen pasos similares para la concreción de un producto creativo que sean aplicables a cualquier individuo que haya producido algún objeto innovador y original. La realidad apunta a que cada individuo tiene su propia concepción de lo que es un desarrollo de esa naturaleza. Sin embargo, a pesar de que cada individuo tiene su forma de trabajar, según Mihaly Csikszentmihalyi (1996),existen hilos conductores que podrían constituir lo que llamamos fases para la concreción de un producto creativo (ver Figura 1). 


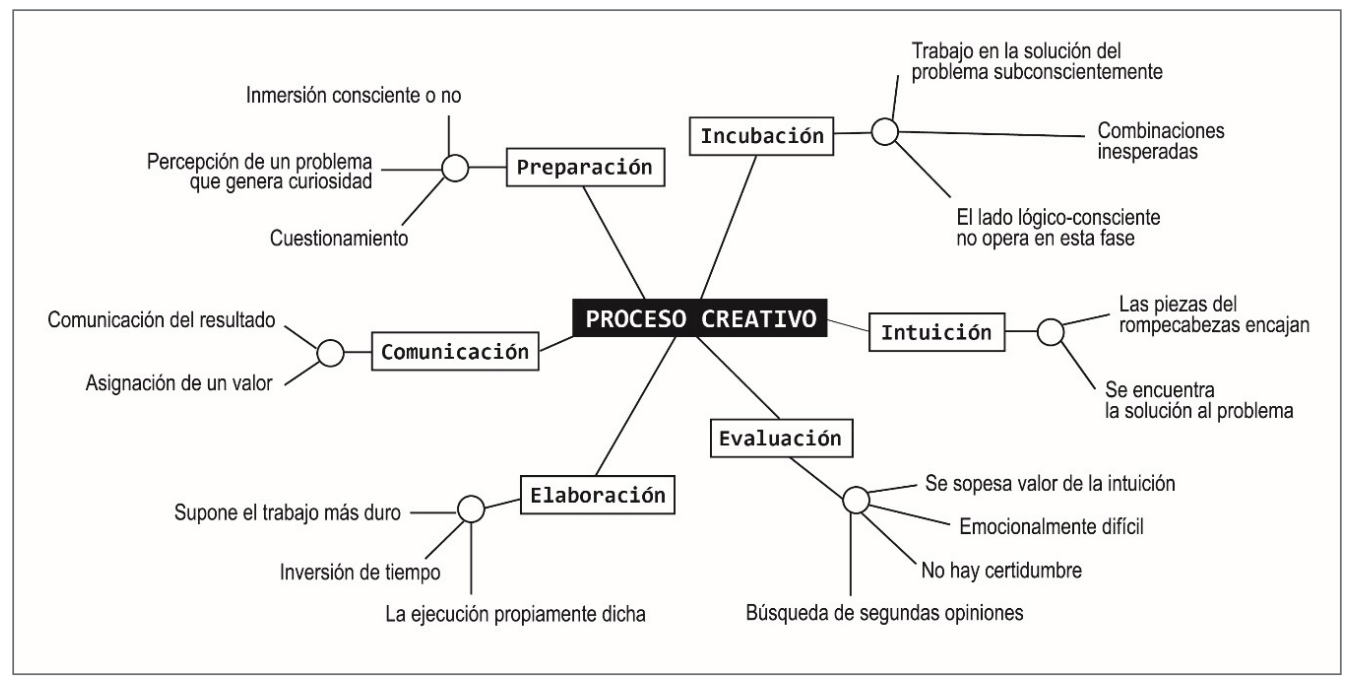

Figura 1: Mapa Conceptual de Las fases del proceso de creación (Ilustración de la autora).

Es importante mencionar, que las fases del proceso creativo no son lineales. Su orden no puede ser tomado literalmente: los procesos creativos reales se desarrollan de forma intercalada, se puede estar en la etapa de la evaluación y durante la misma tener intuiciones, incluso durante la incubación. O también, como se ha constatado en la presente investigación, un sólo proyecto puede desencadenar otros proyectos, por lo que se tiene la impresión de que no hay conclusión sino que son flujos de trabajo constantes - dependiendo del tema-, por lo que puede haber períodos de incubación que duren años.

De cualquier manera, la visión del proceso creativo en seis etapas, aunque demasiado simplificada, ofrece una forma válida y simple de organizar lo complejo que puede llegar a ser dicho proceso. Las siguientes fases son la conjunción de la visión de los expertos en procesos creativos: Mihaly Csikszentmihalyi (1996), Manuela Romo (1997) y el mexicano Mauro Rodríguez Estrada (1985), que se han unido porque se considera son complementarias. En el siguiente mapa conceptual, se ejemplifican estas fases a través de sus aspectos clave:

En cuanto a los métodos de producción de ideas utilizados en el Arte Digital Mexicano (en adelante ADM), tenemos que son distintos a los métodos producidos en el arte tradicional, ya que presentan distintos condicionantes que hacen factible que otros procesos sean viables en este ámbito. Tales condicionantes son: el uso de dispositivos electrónicos, el uso de diversas fuentes de 
energía y el uso del ordenador dentro del proceso o en la obra misma; lo que exige cierta planificación previa al desarrollo de la obra artística. Lo producido en el ADM sería muy difícil que fuera el resultado de una improvisación: las obras investigadas en el ADM para el presente texto presentan procesos de trabajo complejos, en cuanto requieren de fases dentro del proceso de trabajo más puntuales y específicas que exigen, las más de las veces, trabajo colectivo coordinado entre profesionales de varias disciplinas.

Es importante mencionar que una de las características de la mayoría de los artistas del ADM es que en sus inicios produjeron obra desde la austeridad, es decir, sin recursos económicos para producir. Es frecuente ver obras donde los objetos utilizados se encontraron en "mercados de pulgas" o mercados de cosas usadas, como es el caso del desarrollo de Netart "Anima" de Arcángel Constantini (2002), los robots llamados "Parásitos urbanos" de Gilberto Esparza (2006), o los primeros desarrollos de Leslie García ${ }^{1}$, donde se involucra en el surgimiento de las redes de código libre y su formación estuvo permeada por el conocimiento compartido y divulgado en Internet de los tutoriales de programación, entre otros recursos.

\section{ARTISTAS DIGITALES MEXICANOS}

Se han analizado los procesos de creación de artistas digitales mexicanos (algunos de ellos artistas contemporáneos con obra digital), a saber: Arcángel Constantini², Iván Abreu, Gilberto Esparza, Leslie García, Erick Meyenberg, Santiago Itzcoatl, Roberto Morales Manzanares y Rafael Lozano-Hemmer ${ }^{3}$ (residente éste último en España, Canadá y México). basados en las fases del proceso creativo a través de cuestionarios directos aplicados en entrevistas personales y vídeo conferencias ${ }^{4}$.

Los parámetros de selección de artistas digitales fueron varios. Antes de todo debían ser artistas del arte digital activos a la fecha. Este parámetro se especificó debido a que hay artistas con producción digital cuyo trabajo posterior ha derivado hacia otras formas de expresión artística no digital. Los artistas debían contar con diez años o más realizando obra, un periodo que permite hacer un estudio progresivo de su forma de trabajar, necesario para revisar la continuidad en su trabajo artístico y establecer un análisis de la secuencialidad de la obra. En principio, los artistas analizados debían ser nacidos en México 
o con la mayor parte de su obra realizada en este país,si bien haber nacido o continuar viviendo en México no fueron condiciones para el análisis de su obra: la migración y el nomadismo son un fenómeno cotidiano presente también en los artistas (como Rafael Lozano-Hemmer). Debían contar con obra difundida o reseñada en medios electrónicos e impresos: textos que hablan de su labor en algún medio especializado o reseñas en libros, revistas, notas periodísticas, medios electrónicos...Ello permitió tener disponible tanto la obra realizada como críticas y textos de análisis. Otro aspecto que se consideró en la selección de los artistas fue el haber creado obras reflexivas con posible capacidad transformadora. Es decir, obras artísticas que invitaran a la reflexión sobre aspectos de interés para el artista o una colectividad. A este respecto fue interesante ver que los artistas digitales mexicanos tienen preocupaciones políticas,ambientales, sociales, científicas, además de los aspectos estéticos.

A continuación, los resultados generales de la investigación sobre los procesos de creación de los artistas digitales mexicanos:

Preparación. Se indagó sobre el contexto (o formación previa del artista) cuya incidencia en el proceso es relevante. Se encontró que un entorno cultural rico en diversos estímulos nutre el corpus mental del creador y posteriormente sus ideas. Se observa en los artistas el interés o curiosidad innata hacia diversas áreas del conocimiento humano y no únicamente en lo que se refiere a la parcela del Arte Digital. En apariencia, los artistas parecieran no estar excesivamente atentos a su ámbito, y sí a los diversos descubrimientos científicos y tecnológicos. Lo contrario es lo cierto: ellos se han convertido en investigadores de aquello que encuentran con valor y a la postre satisface su curiosidad.

Los intereses comunes en esta fase están en la música, la lectura de textos diversos, la intención de humanizar la tecnología,el encontrar paralelismos históricos, la percepción del arte como proceso de conocimiento que posibilite el cuestionamiento... pero también la reflexión social. La mayoría de los artistas indican tener registro de sus ideas, las cuales conservan y hacen uso de las mismas durante de los procesos de creación.

Uno de los hallazgos más importantes, acaso sea la detección del trabajo artístico en "Procesos de trabajo continuos", que ramifican en diversos sub proyectos que posiblemente devengan en apéndices u obras. Así como la vena intelectual que los artistas de este estudio poseen, por tanto, se afirma que no puede haber creación artística sin reflexión intelectual y/o la búsqueda de la misma a través de la obra. 
Intuición e Iluminación. Encontramos que las buenas ideas no surgen de manera fortuita, sino que son producto del tiempo y de la formación del artista, un proceso que, en todos los casos ha tomado varios años (los artistas estudiados tienen más de 10 años de trabajo artístico). En este sentido, la calidad de las ideas no es aleatoria, deben su aparición a la formación, a la fase de preparación y al contexto del artista. Considerando lo anterior, también se hace uso de recursos facilitadores para la generación de ideas, los artistas del ADM mencionan el sueño, la meditación y la inducción a un alto nivel de concentración a través de distintos medios.

Los artistas estudiados encuentran inspiración en la ciencia, la tecnología, la filosofía, la ciencia-ficción, las tecnologías obsoletas, la política, en aspectos de índole social como la injusticia, en el arte mismo - de manera general, aunque no exclusivamente-.Acuden a la fantasía o ficción, de la misma forma que a la contemplación de su realidad nacional, tecnológica, cultural y social. Algunas de sus ideas surgen de manera rápida, como ocurrencias, pero otras toman años en surgir.

Se observa hacen uso de los principios ya estudiados en la teoría de la creatividad de forma intuitiva, tales como la combinación, la asociación y el retomar elementos ajenos al tema para propiciar abordajes originales en torno a los mismos, produciendo asociaciones "forzadas" que alejan los resultados de soluciones trilladas o comunes.

Evaluación. Es común en todos los casos la fascinación por el proceso de adquisición de conocimiento (producido y adquirido) en sí, más que por el proceso de creación de una obra. Como característica general, se observa que en las obras los resultados son -en todos los casos- indeterminados, no previsibles. Los procesos de investigación y conocimiento que inician los artistas pueden durar meses o años y no necesariamente dar como resultado una obra artística. Cuando estos procesos derivan en una obra, estas no constituyen el fin del trayecto, sino posiblemente el comienzo de otros más. Por lo que el trabajo está concatenado y la valoración de una idea: si es viable de ser realizada o no, es compleja, toda vez por que la idea inicial ha quedado atrás en el tiempo o simplemente porque el artista sabe que eso es lo que hay que realizar.

Los artistas analizados realizan la evaluación de las ideas por intuición, pero siempre responden a cuestionamientos personales o colectivos. En otros casos la factibilidad de que una idea sea llevada a cabo o no, depende de 
aspectos técnicos y limitantes tecnológicas, como también a la falta de recursos económicos para que sea producida. Es frecuente en esta etapa que los artistas socialicen sus ideas con colegas o colaboradores para saber si es factible su realización.

Elaboración. Se observa que las decisiones en torno al perfeccionamiento o adaptación de una obra (en aspectos técnicos o en relación a su ubicación, etcétera) se llevan a cabo -las más de las veces- con grupos de colaboradores (no así la ideación, que en los casos estudiados la realiza el artista). Por ello, la idea inicial de la elaboración de una obra en solitario, la mayoría de las veces, desaparece en este esquema de realización de las ideas dentro de grupos interdisciplinarios.

Los artistas del ADM analizados hacen énfasis en el trabajo intenso físico y mental que conlleva la elaboración de las obras. Los artistas de este ámbito en específico poseen una gran capacidad para el trabajo interdisciplinario y dentro de equipos de trabajo con profesionales de diversas áreas del conocimiento, donde la obra permanece abierta o indeterminada hasta el final. La duración de las mismas es variable, los artistas comentan que una obra les puede llevar horas, semanas, meses o hasta años.

Comunicación. Hay una preocupación por la reacción del público, es decir, la forma en que el espectador participa con la obra es parte de los aspectos que el artista considera al realizarla; incluso en aquellos casos que se deja la obra abierta, entre cuyas posibilidades intrínsecas el público da la "forma final" a la obra o en casos donde la pieza ofrece "finales" variados. A través de la obra el artista busca, a veces, la reacción, a veces, la interacción, donde por lo regular surge lo inesperado.

En este sentido, es importante mencionar que la falta de espacios de exhibición y de apoyos, aunados al desconocimiento del ámbito del ADM, hacen difícil el crecimiento en el número de artistas. No obstante, el interés es creciente. En una plática que sostuvimos con Christa Sommerer en el Taller Solar Insects que impartió en el Laboratorio Arte Alameda en la Ciudad de México en 2013, preguntó a la investigadora responsable de este proyecto si consideraba que en México hubiera un boom o auge en el Arte digital, a lo que se le respondió que sí. No obstante, esto solo afecta a algunas ciudades del país como Ciudad de México, Guadalajara, Monterrey, Puebla, San Luis Potosí, Tijuana, León, y otras que cuentan con centros y/o eventos vinculados al Arte digital. En la mayor parte del país sigue habiendo un predominio de las artes tradicionales 
en la práctica artística (la pintura, la escultura y el grabado), ya que, aun y cuando el arte con el uso de tecnología está más difundido, esto no aplica de igual forma para el Arte digital.

A manera de colofón se infiere que la diferencia del Arte digital producido en México y el realizado en otros países radica en los temas abordados por los artistas. Si bien el proceso es muy similar al observado en otras naciones - como en Brasil donde se elaboran obras de Arte digital con el mínimo de recursos-, es en los temas abordados donde se analizan de manera crítica la idiosincrasia y cosmogonía mexicana. Por citar algunos ejemplos, obras tales como“Arma Sonora Telemática" (2012, Leslie García y Astrovandalistas), una herramienta para realizar manifestaciones en el espacio físico a través de redes sociales, aborda el rol de la violencia en la historia reciente de México y el 2 de octubre del 68; "Voz Alta" (2008, Rafael Lozano-Hemmer), memorial para el 40 aniversario de la masacre de estudiantes en Tlatelolco, donde los participantes comparten en un megáfono lo que vivieron ese día, al tiempo que el propio megáfono controla cuatro luces buscadoras que se proyectan en el cielo y lo expresado se transmite en vivo en la radio"; "Emperadores desplazados" (1997, Rafael Lozano-Hemmer), Instalación que transforma el Castillo Habsburg en Linz, Austria, con proyecciones de una mano, el interior del Castillo de Chapultepec en México, el penacho de Moctezuma (a la fecha en Austria), seguidas por las joyas del imperio Austrohúngaro y música de la cantante mexicana Toña la Negra"; "El auténtico Chilango" (2002, Arcángel Constantini), desarrollo en Internet que muestra una pecera con un axolote (salamandra autóctona ${ }^{7}$ ):la pecera se ubicó en el transporte colectivo metro, la cual es observada por cientos de usuarios, y el artista establece una metáfora entre el axolote y el chilango (como se le llama a los habitantes de la Ciudad de México), ya que ambos dependen de un entorno común cada vez más vulnerable, que anticipa el agotamiento de los recursos naturales para ambas especies y su posible extinción.

De alguna forma las obras anteriores, entre otras producidas en el Arte digital mexicano, muestran el sincretismo cultural que nos ha caracterizado a lo largo de la historia prehispánica, colonial y postcolonial. Tales características son únicas e irrepetibles en todo el orbe. 


\section{CONCLUSIONES}

La presente investigación contribuye a la formación de conocimiento en torno a artistas digitales contemporáneos mexicanos y sus procesos de producción, ya que a la fecha no existen documentos virtuales o físicos al respecto con la perspectiva que aquí se le imprime. Se han estudiado los procesos de reflexión y conceptualización en torno al trabajo artístico, desde el punto de vista de la realización, así como del proceso intelectual llevado a cabo por el artista. Se ha hecho una reflexión sobre estos nuevos procesos de producción en el Arte Digital Mexicano, sus fuentes de alimentación y cómo han transformado la práctica artística del ámbito.

Se ha hecho una propuesta metodológica interdisciplinar derivada del estudio y análisis de los procesos de producción de artistas digitales mexicanos, la cual se incluye en una investigación más profunda sobre el tema (Villagómez 2017).

Sea pues, que el lector haya encontrado parte de la explicación y razones al otrora considerado misterioso proceso de la creación artística, así como dilucidado los vínculos con una parte importante y fundamental del escenario del Arte Digital del México de nuestros tiempos.

\section{Referencias}

Benjamin, Walter. (1936) 2003. La obra de arte en la época de su reproductibilidad técnica. Traducción Andrés E. Weickert. México: İtaca. http://artes.lapiedrahita.com/wp-content/ uploads/2014/07/La-obra-de-arte-en-la-era-de-la-reproductibilidad-te\%CC\%81cnica.pdf

Csikszentmihalyi, Mihaly. (1990) 2007. Fluir (flow) Una psicología de la felicidad. Traducción de Nuria López. Barcelona: Kairos

- . 1996. Creatividad, el fluir y la psicología del descubrimiento y la invención. Traducción de Pedro Tosaus Abadía. Barcelona: Paidós

Deleuze, Gilles \& Félix Guattari. (1980) 2009. Rizoma. Traducción David A. Rincón. México DF: Fontamara

Esteve de Quesada, Albert. 2002.Creación y proyecto: El método en diseño y otras artes. Valencia: Institució Alfons el Magnani. https://es.scribd.com/document/115156987/Creacion-y-proyecto-Capitulo-3-Albert-Esteve-de-Quesada

Gardner, Howard. 1995. Mentes creativas una anatomía de la creatividad. Traducción de José Pedro Tosaus Abadía. Barcelona: Paidós

Hernández Sampieri, Roberto, Carlos Fernández Collado \& María del Pilar Baptista Lucio. (1991) 2010. Metodología de la investigación. México DF: McGraw-Hill Interamericana 
Lieser, Wolf. 2009. Arte digital: Nuevos caminos en el arte. Con colaboración de Tilman Baumgärtel... et al.; traducción, KatinkaRosés Becker. Colonia: H.F. Ullman

Malvido, Adriana. 1999. Por la vereda digital. México DF: Conaculta

Marina Torres, José Antonio. 2004. Elogio y refutación del ingenio. Barcelona: Anagrama

— . 2006. Teoría de la inteligencia creadora. Barcelona: Anagrama

Maslow, Abraham Harold. (1963) 2005. La personalidad creadora. Traducción: Rosa Ma. Raurich. Barcelona: Kairos

Paul, Christiane. 2003. Digital Art. London: Thames \& Hudson

Popper, Frank. 1993. Art of the electronic age. Translated from the French by Bernard Hemingway.New York: Thames\& Hudson

Rodríguez Estrada, Mauro. 1985. Manual de Creatividad. Los procesos psíquicos y el desarroIlo. México DF: Trillas

Romo Santos, Manuela. 1997. Psicología de la creatividad. Barcelona: Paidós

Rush, Michael. 2005. New media in Art. Londres: Thames \& Hudson

Shanken, Edward. 2009. Art and electronic media. London: Phaidon

Villagómez Oviedo, Cynthia Patricia. 2010. Métodos de la creatividad aplicados al diseño gráfico y las artes visuales. Guanajuato: Universidad de Guanajuato

— . 2017. "Análisis de procesos de producción artística digital en México: artistas digitales mexicanos y su obra". Tesis Univ. Politéctica de València. www.lalibreria.upv.es/portalEd/ UpvGEStore/products/p_5836-1-1

Wands, Bruce. 2007. Art of the digital age. Nueva York: Thames \& Hudson

Zinker, Joseph 2004. El proceso creativo en la terapia guestáltica. Traducción, Luis Justo. México DF: Paidós

\section{Notas}

1 Disponible en: https://astrovandalistas.cc/ast/

${ }^{2}$ Disponible en: http://www.unosunosyunosceros.com/EAC/time.htm

${ }^{3}$ Disponible en: http://www.lozano-hemmer.com/projects.php

${ }^{4}$ Entrevistas realizadas por Cynthia Villagómez, Consultables en línea como material complementario en https://vimeo.com/user29545447: Iván Abreu en la ciudad de León, Gto., México. 13 de diciembre 2012, Galería Jesús Gallardo, dentro del marco del "Encuentro de Arte de León", en el ciclo de conferencias "Todo lo que quieras saber de Arte contemporáneo antes del 21 de diciembre", donde participó en la mesa de trabajo: "Lo virtual, plataforma flotante", llevado a cabo del 13 al 15 de diciembre de 2012. Duración 00:32:43. I Entrevista a Arcángel Constantini en la Fonoteca Nacional, Coyoacán, Ciudad de México. 1 de octubre de 2011. Duración entrevista en video 00:21:43. / Entrevista a Gilberto Esparza en el estudio del artista en la Ciudad de México, 30 de julio de 2012. Duración 00:42:15. / Entrevista a Leslie García (vídeo conferencia), 15 de enero de 2013. Duración 01:09:41. / Entrevista a Roberto Morales Manzanares (vídeo conferencia), el 4 de septiembre 2012. Duración 00:36:52. / Entrevista a Santiago Itzcóatl (vídeo conferencia), 16 de enero 2013. Duración 00:59:25. / Entrevista a Erick Meyenberg (vídeo conferencia), 20 de mayo 2014. Varias secuencias de vídeo con duración variable. 
${ }^{5}$ Cabe mencionar que lo sucedido el 2 de octubre del año 68, hasta hace dos décadas no se comentaba en los medios de comunicación, era un secreto a voces.

${ }^{6}$ En México es motivo de encono que un símbolo nacional de esta envergadura se encuentre en otro país.

7 Tipo de salamandra que se encuentra únicamente en los canales de Xochimilco en la Ciudad de México, una especie actualmente amenazada que fue de amplia veneración en el mundo prehispánico).

(Artículo recibido 05-03-18; aceptado 08-05-18) 